\title{
Transformation strain based method for characterization of convective heat transfer from shape memory alloy wires
}

\author{
Anupam Pathak, Diann Brei and Jonathan Luntz \\ Mechanical Engineering, University of Michigan, 2250 GG Brown, Ann Arbor, \\ MI 48109-2126, USA \\ E-mail: dibrei@umich.edu
}

Received 10 July 2009, in final form 29 November 2009

Published 2 February 2010

Online at stacks.iop.org/SMS/19/035005

\begin{abstract}
While shape memory alloys (SMAs) have many actuation benefits, their frequencies are commonly restricted by slow cooling times caused by limitations in convective heat transfer. To increase the cooling speed and at the same time reduce excess power consumption from overheating, it is critical to understand the heat transfer from SMA wires. This requires accurate surface temperature measurement under a fixed input power, which is difficult to obtain using traditional methods because of the nature of SMAs (thin wires, large strains, heat activation, ambient environment, etc). This paper introduces a non-invasive technique for calculating the convective coefficient for SMAs by employing the temperature-induced transformation strain of SMAs to estimate the surface temperature. This method was experimentally validated for measurement of the convective coefficient in air where infrared cameras can operate, and then used to indirectly measure the convective coefficient across a range of commonly utilized SMA wire diameters and ambient media where traditional methods are limited. Formulated empirical correlations to the collected data provide a mathematical relationship to calculate the convective coefficient in material models which serve as better estimates of convection, and may be used for optimization of SMA actuators for increased frequency performance while ensuring that power draw is minimized.
\end{abstract}

(Some figures in this article are in colour only in the electronic version)

\section{Introduction}

Since their discovery, shape memory alloys (SMAs) such as Nitinol have been used in a diverse range of applications that take advantage of the high energy density, large actuating strain, and extremely low weight and size of such materials [1-4]. When subjected to a temperature change, SMAs undergo a change in their internal crystal structure (between martensite and austenite) resulting in considerable recoverable strain that produces usable work. Typically, SMAs are employed as actuators by electroresistively heating the material, and then cooling and deforming the material under a lower stress to close the work cycle. This mode of SMA operation can be found in many existing applications in areas requiring vibration cancelation [5-8], aerodynamic flow control [9-11], robotics/biomimetics [12-15], micropumps/valves [16-19], and micro-grippers [18, 20-24].

While the internal transformation at the atomic-lattice scale of a SMA is fast-of the order of the elastic shear wave speed [25]—observed transformation rates are usually much slower due to limitations in the rate of heat transfer. Heating the wire can be achieved rather quickly, of the order of milliseconds through electro-resistive heating [26]. However, the cooling cycle presents significant speed limitations [27] because cooling is done passively and is limited by the SMA wire's surface area to volume ratio, geometry, and the convective properties of the ambient medium [28]. The cooling time of a SMA can be improved by altering the properties of its cooling medium; yet, this comes with a penalty of increased heat losses and thus power draw during the heating cycle [29]. Decreasing the diameter of the actuator wire, which 
increases the surface to volume ratio and facilitates ambient fluid flow, can also produce faster responses, but this decreases the actuator's force output. Understanding these tradeoffs is crucial, since over-specifying the cooling of an actuator may employ wires of insufficient diameter producing inadequate force or use a medium with unnecessarily high convective properties leading to excess power consumption. Underspecifying the cooling can result in limitations in cooling speed, which limit the actuator's frequency capabilities.

Thus, to optimize a SMA during dynamic cycling it is important to be able to predict the effects of heat transfer for various wire diameters and environmental fluids. Unfortunately, this is complicated since commonly used SMA wires are extremely thin (e.g. diameters below 20 mil, or about $0.5 \mathrm{~mm}$ ) and measuring their surface temperatures to calculate the convective coefficients is challenging. With thicker samples of conventional materials, temperature measurements are achieved by mounting thermocouples directly on the surface. For example, experiments for long cylinders [30, 31] have been accomplished using surface-mount thermocouples on large diameter samples (e.g. $28 \mathrm{~mm} \mathrm{[32])} \mathrm{to} \mathrm{monitor}$ the cylinder surface temperature $\left(T_{\mathrm{s}}\right)$ for a fixed ambient temperature $\left(T_{\mathrm{a}}\right)$ and applied power $P$. Knowing the cylinder surface area $A$, Newton's law of cooling, neglecting radiation [33] due to relatively low surface temperatures,

$$
P=h A\left(T_{\mathrm{s}}-T_{\mathrm{a}}\right)
$$

is applied to solve for the convective coefficient $h$. This method is typically not possible with a SMA because it is difficult to mount thermocouples to the smaller diameter wires and to keep them connected over the large strains (4$8 \%$ ) of the SMA during temperature-induced transformation. Thermocouples are also invasive since heat transfer occurs from the SMA sample to the thermocouple through its bonding agent reducing the measured surface temperature. This effect is enhanced for the smaller diameter SMA wires that are of the order of some of the smallest commercially available thermocouples [34]. Using resistivity to predict temperature in a SMA is also difficult since it is non-monotonic with temperature and changes with cyclic history [35-37]. Direct surface temperature measurement using infrared cameras is often used for convective studies [38], though this method is difficult for very thin SMA wires due to resolution limitations. Additionally, capturing thermal images is extremely difficult in environments other than air (e.g. thermal grease, oil, and water) [39]. The issue of temperature measurement is further complicated since the convective coefficient is known to differ depending on the surface properties (roughness, temperature) of a particular material, making the use of conventional materials such as tungsten, used in hot wire anemometers, infeasible. Kandlikar et al [40], for example, reported a $40 \%$ increase in heat transfer for laminar flow (a 70\% increase for developing flow) when doubling the average surface roughness from $1 \mu \mathrm{m}$ in a $0.62 \mathrm{~mm}$ diameter tube, and concluded that the effect will be more pronounced for decreasing geometries. Indeed, on the microscale Koo and Kleinstreuer [41] predict a $25 \%$ increase in convection for just a $4 \%$ increase in relative roughness. Depending on the manufacturing process, the surface roughness of the SMA oxide layer can appreciably differ in the characterized ranges. For example, in a study by Pohl et al [42], average roughness was shown to decrease from 0.8 to $0.2 \mu \mathrm{m}$ through electrolytic polishing. Therefore, it is crucial that the convective coefficient is measured for a particular material of interest (especially for small geometries), as the use of another material has a high potential to introduce error. For general estimates of the convective coefficient, there are non-dimensionalized empirical relationships in the literature $[30,31]$ that may be extrapolated to smaller SMA wire diameters-between 6 and $20 \mathrm{mil}(0.15-0.51 \mathrm{~mm})$. However, even at the larger diameters on which the models were based $(28 \mathrm{~mm})$, errors of up to $20 \%$ [33] are typical, which is generally not acceptable when accurate predictions of speed and power draw are required.

To address these challenges, this paper introduces a noninvasive technique - the transformation strain based methodto calculate the convective coefficient, $h$, for a SMA by taking advantage of the material's temperature-induced transformation strain to estimate the surface temperature $T_{\mathrm{s}}$. With this estimate, Newton's law of cooling (equation (1)) can be utilized to calculate the convective coefficient, $h$, by immersing a wire in a temperature-controlled medium (fixed $T_{\mathrm{a}}$ ) and monitoring the steady-state power $P$ applied to the wire of known surface area $A$. This new experimental method can be directly applied to any size SMA material of interest (including microscale) in any ambient environment, whereas methods such as standard infrared imaging techniques can only be applied accurately in air for thick wires. To validate the method, experiments were first conducted in air with thick wires so that they could be compared to those obtained using a standard infrared imaging technique for large diameter wires. The experiments were then extended for a range of commonly used smaller commercial wire diameters in a variety of ambient environments where most standard techniques do not apply: still air, flowing air (of varying flow rate), mineral oil, thermally conductive grease, and water. Empirical correlations based upon physical variables such as wire diameter, fluid properties, temperature and flow rate, were formulated by fitting correlations from the literature [33] and optimizing the free variables. These correlations provide continuous relationships to estimate convective coefficients within and beyond the tested regime which engineers can use for computational optimization of SMA actuators, ensuring that cooling requirements are met and that power draw is minimized.

\section{Transformation strain to temperature correlation}

To accurately calculate the convective coefficient, $h$, of a SMA, the transformation strain method uses the Newton's law of cooling (equation (1)), but the surface temperature is not directly measured. Instead, the surface temperature is mapped to the midpoint of full strain recovery. A correlation between the midpoint transformation strain and the surface temperature for each wire sample is obtained through a series of very slow (quasi-static) heating cycles in an environmental chamber under near-zero loads. This correlation is used to estimate 


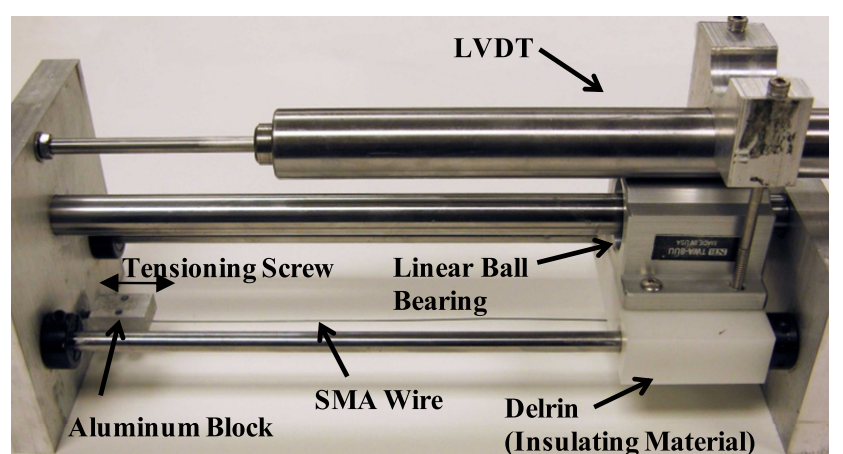

(a)

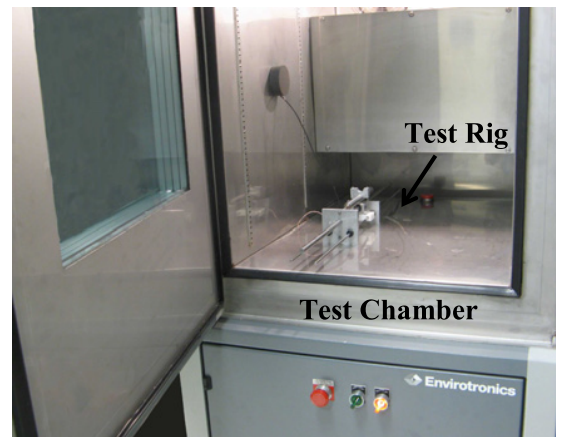

(b)

Figure 1. Test apparatus for midpoint transformation temperature measurements. The temperature of a length of SMA wire is precisely controlled by slowly varying the temperature in the environmental chamber while its length is measured using a LVDT attached to a linear bearing. (a) Experimental test apparatus. (b) Apparatus in an environmental chamber.

the surface temperature $T_{\mathrm{s}}$ in all the convective coefficient experiments in this paper.

To obtain the midpoint transformation temperatures for the SMA wires, each sample was heated in an Envirotronics FLX-300 environmental chamber while recording strain. For each test, a 7 inch sample of the widely used $70^{\circ} \mathrm{C}$ Flexinol (Dynalloy) SMA was installed in the test setup (figure 1) with one end of the SMA connected to a very low friction sliding stage that moves when the SMA is heated, and the other end attached to an aluminum block fixed by a tensioning screw. The free austenite reference length was set by heating the wire and adjusting the wire tension through the tensioning screw until all slack was taken out. A residual tension remained in the wire to offset the very small friction in the linear ball bearing. The wire was then allowed to cool and was strained with the tensioning screw to a $4.5 \%$ elongation from its austenite free length (figure 1(a)). The ambient temperature inside the chamber was slowly increased (several minutes per degree near transition) to ensure proper mixing. Motion recovery occurred rapidly (over only $1{ }^{\circ} \mathrm{C}$ for all wires), and since motion did not occur $1{ }^{\circ} \mathrm{C}$ below this recovery temperature, the chamber was assumed to hold a relatively constant temperature since any fluctuation would have triggered transformation. In the experiment, motion recovery was measured with a linear variable differential transformer (LVDT) sensor monitored by an oscilloscope and the midpoint transformation temperature was recorded when the slider position was half way between the $4.5 \%$ prestrain and the austenite free reference length.

The procedure was repeated twice for each different wire diameter (ranging from 6 to $20 \mathrm{mil}$ ) and two separate spools of 10 mil SMA wire were also tested. It was assumed that due to these near-zero loads, and small reset loads, that the wire's material properties did not change over many cycles [37, 43]. The measured midpoint transformation temperatures (table 1) varied slightly for the different wire diameters, ranging from $57-63^{\circ} \mathrm{C}$ with an average deviation of $2.8 \%$ from the mean of $61.2^{\circ} \mathrm{C}$. A higher deviation was observed spool to spool. For example, when $10 \mathrm{mil}$ (about $0.25 \mathrm{~mm}$ ) wires from two different spools were tested, their deviation from one another was $4.8 \%$. However, testing different wire segments taken from the same spool produced constant and
Table 1. Measured midpoint transformation temperatures. Temperatures were measured at a midpoint strain of $2.25 \%$ for $70^{\circ} \mathrm{C}$ Flexinol SMA wire under near-zero load and $4.5 \%$ initial strain.

\begin{tabular}{|c|c|c|c|}
\hline $\begin{array}{l}\text { Spool } \\
\text { number }\end{array}$ & $\begin{array}{l}\text { Diameter } \\
\text { (mil) }\end{array}$ & $\begin{array}{l}\text { Midpoint } \\
\text { transformation } \\
\text { temperature } T_{\mathrm{s}}\left({ }^{\circ} \mathrm{C}\right)\end{array}$ & $\begin{array}{l}\text { \% Deviation from } \\
\text { average midpoint } \\
\text { transformation } \\
\text { temperature }\end{array}$ \\
\hline 1 & 6 & 61 & +1.2 \\
\hline 2 & 7 & 59 & -2.1 \\
\hline 3 & 10 & 59 & -2.1 \\
\hline 4 & 10 & 62 & +2.8 \\
\hline 5 & 12 & 57 & -5.5 \\
\hline 6 & 15 & 61 & +1.2 \\
\hline 7 & 20 & 63 & +4.5 \\
\hline
\end{tabular}

repeatable results within less than $1 \%$ error, which is within the accuracy of the test chamber. Thus, the transformation temperature is not highly dependent on wire diameter but is sensitive to each spool of wire which has its own individual processing history. Therefore, to ensure accuracy in all further experiments, tests representing a particular SMA wire diameter were all performed from the same spool possessing consistent processing history.

\section{Transformation strain based experimental method}

The convective coefficient $h$ can be indirectly calculated using Newton's cooling law (equation (1)) by (1) knowing the cross sectional area $A$ and ambient temperature $T_{\mathrm{a}}$, (2) measuring the applied power with current and voltage probes and (3) correlating the measured midpoint transformation strain to estimate the surface temperature $T_{\mathrm{s}}$. For simplicity, it is assumed that the wire diameter does not change with transformation. Assuming isochoric properties and a 2.25\% motion recovery this assumption produces only $1 \%$ error in surface area. For the experimental method, the SMA samples were tested under varying steady-state resistive heating in air (at various flow rates), still oil, thermally conductive grease, and still water. This combination of media and wire diameter selections was intended to span a wide range of cooling conditions. As validation the convective coefficient 


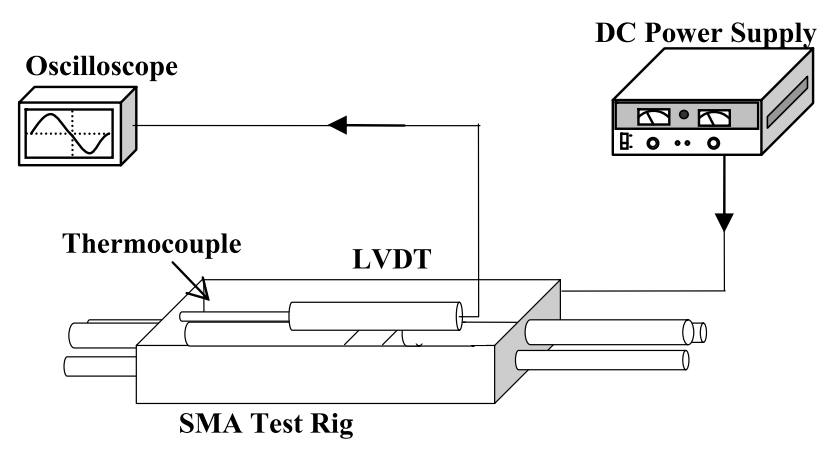

(a)

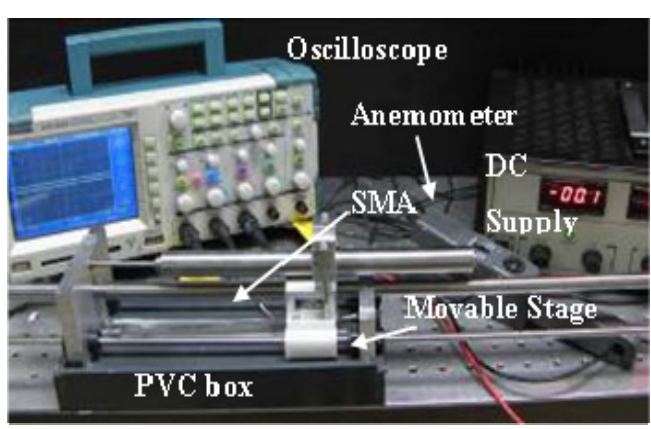

(b)

Figure 2. Experimental test apparatus for $h$-value testing. The leak-proof PVC box is filled with various tested fluids and the strain is monitored while applying electrical power, which is recorded at the midpoint transformation strain. (a) Schematic of experimental setup. (b) Photograph of experimental testing in liquid.

was measured using surface temperature readings with an IR camera for the only two feasible test conditions due to wire size and medium-15 and 20 mil SMA wire in air. Traditional empirical models [33] were used with free parameters $C$ and $m$ optimized using a generalized reduced gradient (GRG) to provide a best fit to the data. These correlations provide a mathematical relationship to calculate the convective coefficient in material models or continuous optimization algorithms.

\subsection{Experimental procedure}

Each test was conducted with the same experimental apparatus used in the midpoint transformation temperature measurements; however, the wire was electro-resistively heated via a DC power supply (figure 2) instead of an environmental chamber. Input power to the wire was computed from the applied voltage and current, which were measured with voltage and current probes that were connected to an oscilloscope. The apparatus was placed with the SMA in a horizontal configuration inside a leak-proof PVC box filled with the various environmental fluids and their ambient temperatures were measured by a type-K thermocouple placed inside the box. For the free convection in air tests, the PVC box was covered to ensure that stray air currents did not affect the measurement. Forced convection in air was conducted outside the box, with an electrical fan whose proximity and speed were varied to produce a desired test flow rate that was measured by a hot wire anemometer. Tests were conducted at flow rates of 150, 250, 450, and $625 \mathrm{ft} \mathrm{min}^{-1}$. In all experiments, the input power was slowly increased and recorded when the half way displacement between the $4.5 \%$ prestrain and the austenite zero reference length was recorded by the LVDT. The recorded power along with the associated ambient temperature and midpoint transformation temperature (table 1) were used in Newton's law of cooling, equation (1), to predict the convective coefficient, $h$. For each wire diameter listed in the table of measured midpoint transformation temperatures (table 1), the test was repeated for different media: in air (free and forced), mineral oil, thermal grease, and distilled water (figure 3 ).

\subsection{Convection in air}

There exists a trade-off between simplicity and performance for cooling in air. For free convection (cooling in still air), no additional complications are introduced to the system, though the convection heat transfer is low. To improve convection, flowing air may be introduced through a fan, producing forced convection. While this often requires additional power draw and system complications, the resulting heat transfer improvements may be sufficient for the speed and power requirements of a given application. This section studies both free and forced convection for SMA wires of varying diameter to provide a better understanding of the heat transfer effects under these regimes.

3.2.1. Free convection in air. In the experimental results for cooling in still air (figure 3) a significant dependency on wire diameter is evident. Overall, the convective heat transfer coefficient decreases as the wire diameter is increased-for 6 mil wire, the $h$-value is $153 \mathrm{~W} \mathrm{~m}^{-2} \mathrm{~K}^{-1}$, while the $h$-value for 20 mil wire is reduced by $44 \%$ to $68 \mathrm{~W} \mathrm{~m}^{-2} \mathrm{~K}^{-1}$. At the upper limit, the $h$-value for the collected data should theoretically approach zero at very large diameters. This is because a very large diameter cylinder would restrict convective flow and the primary mechanism of heat transfer would be through conduction across the fluid. At extremely small diameters the flow would not be resisted and the $h$-value would increase to a 'free-flow' limit.

To validate these results and the general midpoint transformation temperature methodology for measuring $h$, a second experiment was conducted using an infrared camera for surface temperature measurements [38, 44]. Due to focus resolution limitations $\left(\sim 200\right.$ pixels inch $\left.^{-1}\right)$, the IR camera was used to validate measurements for the two largest diameters (15 and 20 mil) in still air since unpredictable inaccuracies would occur when measuring across any airfluid interface. During testing, the SMA wires were clamped in a fixture (figure 4) and heated with an electrical current while the surface temperature was monitored with an infrared imaging system (FLIR ThermaCam SC1000) with a PtSi $256 \times 256$ detector array. During data collection, the SMA 

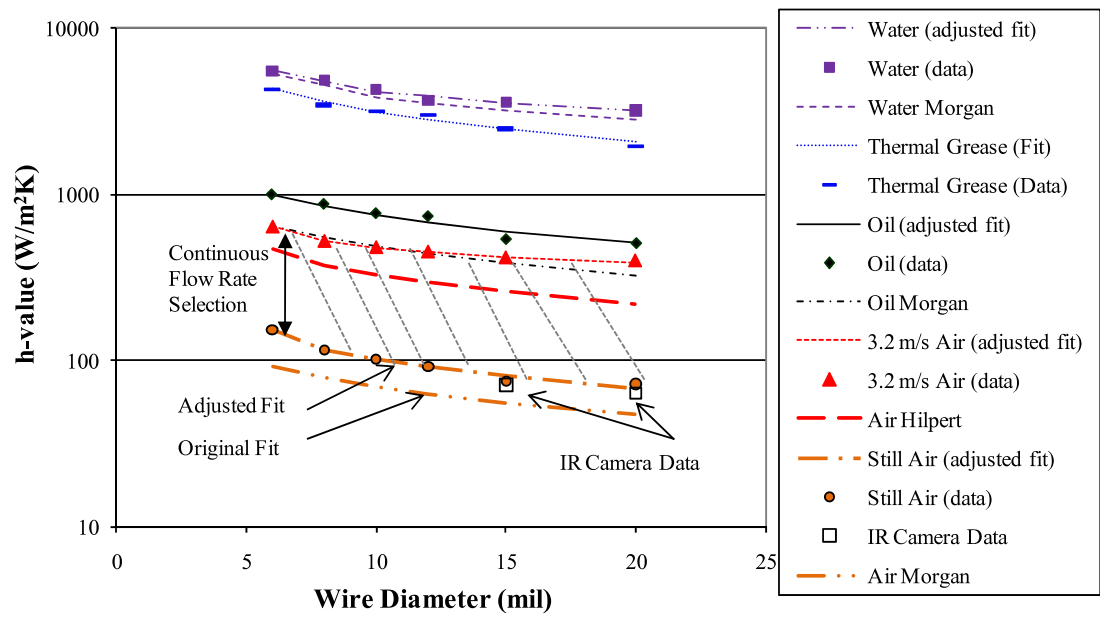

Figure 3. Convective heat transfer coefficients in different media. The measured $h$-value decreases somewhat with wire diameter and varies greatly with cooling medium. The empirical fits plotted with the data closely match the form of the functional dependence. Forced convective air flow range represents continuous space.

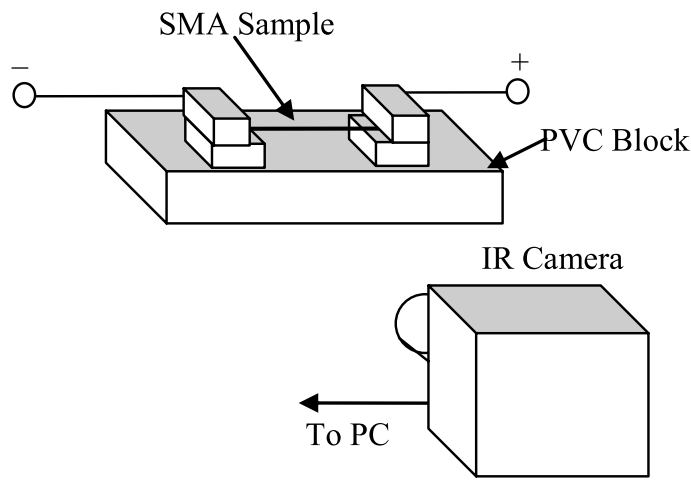

(a)

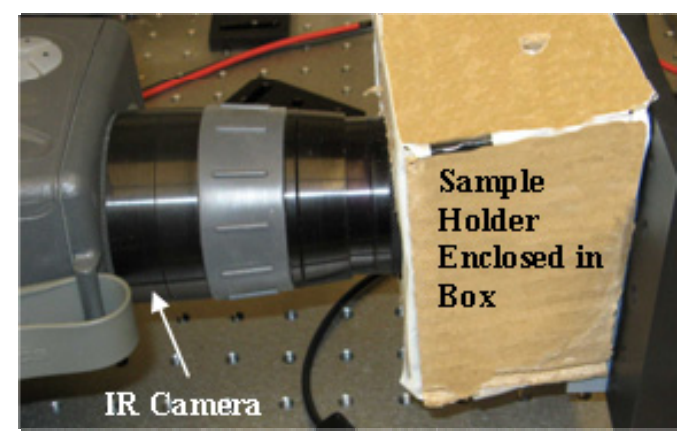

(b)

Figure 4. Experimental test apparatus for IR testing. The SMA sample was shrouded in a box for protection from stray air currents, and an IR camera measured surface temperature under steady-state Joule heating. (a) Experimental setup for IR camera testing. (b) Photograph of testing in progress.

sample was shrouded in a box for protection from stray air currents and the applied current was increased in increments where the steady-state surface and ambient temperatures were recorded. For the surface temperature readings, an emissivity for the $70^{\circ} \mathrm{C}$ dynalloy wire of 0.76 was programmed in the camera. The emissivity was measured by applying a coating of known emissivity to a portion of the wire, heating the sample to a uniform temperature, and comparing readings from the coated and uncoated surfaces. Because for these tests the sample wire length was short $(0.5 \mathrm{in})$, the input power was calculated from the wire resistance and current since the contact resistance of a voltage probe would be invasive. Wire resistivity was measured in both martensite and austenite states for both wire diameters, and the normalized values are listed in table 2 . For temperatures below the transformation temperatures listed in table 1 , the input power was computed using the martensite resistivity, while the austenite resistivity was used for temperatures above the transition. Because the resistivity during transformation is not monotonic and difficult to predict due to its dependence
Table 2. Wire resistivity for SMA wires used in IR testing. Values were measured for austenite and martensite phases for $70{ }^{\circ} \mathrm{C}$ Flexinol.

\begin{tabular}{lll}
\hline $\begin{array}{l}\text { Diameter } \\
(\mathrm{mil})\end{array}$ & $\begin{array}{l}\text { Martensite } \\
\text { resistivity }\left(\Omega \mathrm{m}^{-1}\right)\end{array}$ & $\begin{array}{l}\text { Austenite resistivity } \\
\left(\Omega \mathrm{m}^{-1}\right)\end{array}$ \\
\hline 15 & 8.67 & 7.68 \\
20 & 5.31 & 4.53 \\
\hline
\end{tabular}

on prior cyclic history [37], data were not collected in this temperature range. However, with the data above and below, interpolation could be performed through the transition range.

In figure 3, the two additional data points are plotted for the IR camera measurements, showing excellent agreement. Using the IR camera, the convective coefficient for the $20 \mathrm{mil}$ wire was calculated to be $67 \mathrm{~W} \mathrm{~m}^{-2} \mathrm{~K}^{-1}$ which is only $1.5 \%$ from the measurement from the midpoint transformation temperature method. The 15 mil diameter produced a higher convective coefficient of $73 \mathrm{~W} \mathrm{~m}^{-2} \mathrm{~K}^{-1}$ using the IR camera 
Table 3. Fluid properties for tested media. Properties for air and water were taken from [46], while those for oil were taken from [33].

Thermal grease properties were not known other than the thermal conductivity, listed by the manufacturer [34]. All properties were evaluated at film temperature of $42^{\circ} \mathrm{C}$.

\begin{tabular}{llllll}
\hline & Kinematic viscosity & $\begin{array}{l}\text { Thermal } \\
\text { conductivity } k \\
\left(\mathrm{~W} \mathrm{~m}^{-1} \mathrm{~K}^{-1}\right)\end{array}$ & $\begin{array}{l}\text { Prandtl } \\
\text { number, } \operatorname{Pr}\end{array}$ & $\begin{array}{l}\text { Thermal diffusivity } \\
\alpha\left(\times 10^{6} \mathrm{~m}^{2} \mathrm{~s}^{-1}\right)\end{array}$ & $\begin{array}{l}\text { Thermal expansion } \\
\text { coefficient } \\
\beta \times 10^{3}\left(\mathrm{~K}^{-1}\right)\end{array}$ \\
\hline Fir & $v\left(\times 10^{5} \mathrm{~m}^{2} \mathrm{~s}^{-1}\right)$ & 0.027 & 0.712 & 24.0 & 3.18 \\
Oil & 1.71 & 0.143 & 0.002 & 0.082 & 0.70 \\
Thermal grease & Unknown & 2.300 & Unknown & Unknown & Unknown \\
Water & 0.06 & 0.634 & 4.00 & 0.157 & 0.390 \\
\hline
\end{tabular}

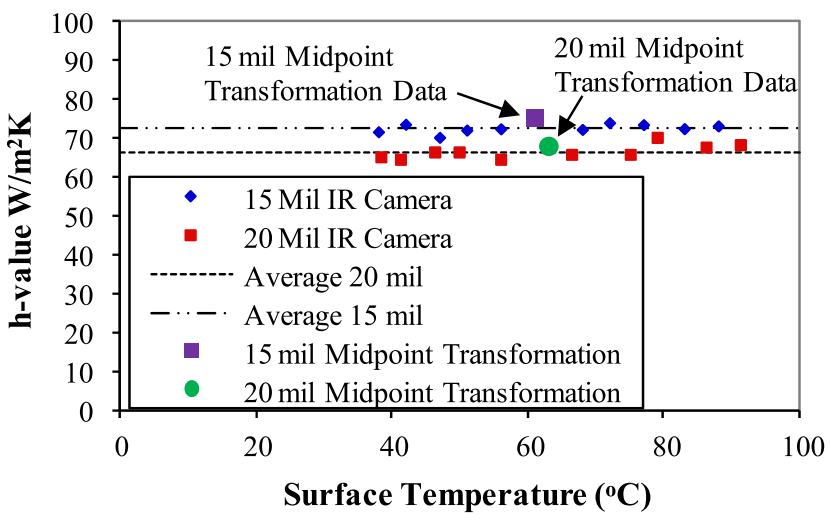

Figure 5. Convection coefficients for free air cooling using an IR camera. $h$-values are independent of temperature well above and below transformation and provide good agreement with midpoint transformation tests.

measurement, which is a $3 \%$ deviation. In figure 5 , the $h$-value measurements using the IR camera are plotted with respect to the wire's surface temperature, and it is evident that the convective coefficient did not vary with temperature in any measurable way. This is probably due to the fact that the properties of air affecting natural convection are not heavily temperature dependent [33]. Therefore, the interpolation of the $h$-value from above and below the wire transformation temperature is valid.

After gathering data for the convective heat transfer in air (assumed to produce the lowest $h$-values), a further calculation was performed to verify the decision to neglect radiation in equation (1). This was performed by examining the ratio of power dissipation between radiation (assuming the StefanBoltzmann law) and natural convection. Assuming a mean cooling surface temperature $T_{\mathrm{s}}$ of $60^{\circ} \mathrm{C}$ (table 1) and a convective coefficient $h$ of $50 \mathrm{~W} \mathrm{~m}^{-2} \mathrm{~K}^{-1}$ (lower bound to the data in figure 4), the ratio in this worst case was calculated to be only 0.09. This shows that convection is indeed dominant, and that neglecting heat transfer due to radiation is a valid assumption.

For the set of data collected for free cooling in air (figure 3), a continuous correlation was formulated using Morgan's correlation for an isothermal horizontal cylinder [30] as a basis because it is well known and has a relatively simple form. In this empirical model, the non-dimensional Nusselt number (ratio of convection to conduction in the fluid) is approximated from

$$
\overline{\mathrm{Nu}}_{D}=\frac{\bar{h} D}{k}=C \operatorname{Ra}_{D}^{m}
$$

where $\bar{h}$ is the average convective coefficient over the entire surface of the wire, $D$ is the wire diameter in meters, $k$ is the thermal conductivity of air (in units of $\mathrm{W} \mathrm{m}^{-1} \mathrm{~K}^{-1}$ ), and $C$ and $m$ are free variables. Individual fits were produced using these free variables for each tested medium, which produced greater accuracy than the single all-encompassing fit originally suggested by Morgan. The Rayleigh number, $\mathrm{Ra}_{D}$, for a cylindrical shape is defined as

$$
\operatorname{Ra}_{D}=\frac{g \beta\left(T_{\mathrm{s}}-T_{\mathrm{a}}\right) D^{3}}{v \alpha} .
$$

In equation (3), $g$ is the acceleration due to gravity, $\beta$ is the thermal expansion coefficient of the fluid, $T_{\mathrm{S}}$ is the surface temperature of the wire, $T_{\mathrm{a}}$ is the ambient temperature of the fluid, $D$ is the diameter of the wire, $v$ is the kinematic viscosity of the fluid, and $\alpha$ is the thermal diffusivity. All fluid properties are evaluated at a film temperature estimated as the average of the surface temperature (the mean of $60^{\circ} \mathrm{C}$, from table 1$)$ and the measured ambient temperature $\left(23^{\circ} \mathrm{C}\right)$, and the values are listed in table 3 . Substituting the Rayleigh number, equation (3), into the Nusselt number correlation, equation (2), and solving for the convection coefficient allows

$$
\bar{h}=k C\left(\frac{g \beta\left(T_{\mathrm{s}}-T_{\mathrm{a}}\right)}{v \alpha}\right)^{m} D^{3 m-1} .
$$

This equation for the average convective coefficient yields additional mathematical insight into the physical limits on diameter. If $3 m$ is less than 1 , which is true for all proposed fits (table 3), $h$ approaches zero for large $D$ and infinity for small $D$. This is in accordance with the limitations of convection due to fluid flow discussed earlier. To optimize the empirical fit, values for $C$ and $m$ were adjusted using a GRG algorithm that minimized the average fit error to the collected data to only $2.4 \%$, which is significantly less than the average $33 \%$ error produced by the original Morgan correlation (figure 3 ). These parameter values are listed in table 4 for the two Rayleigh number regimes that the experimental conditions fell within. Figure 3 shows the empirical fit with the experimentally measured data points. In general, the trend is fitted very well and the equation provides useful values that can be interpolated between two points or extrapolated to diameters that were not measured in the experimental study. 


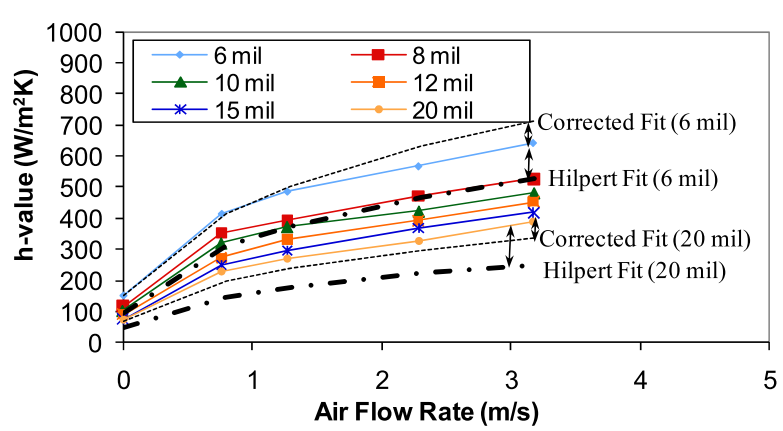

(a)

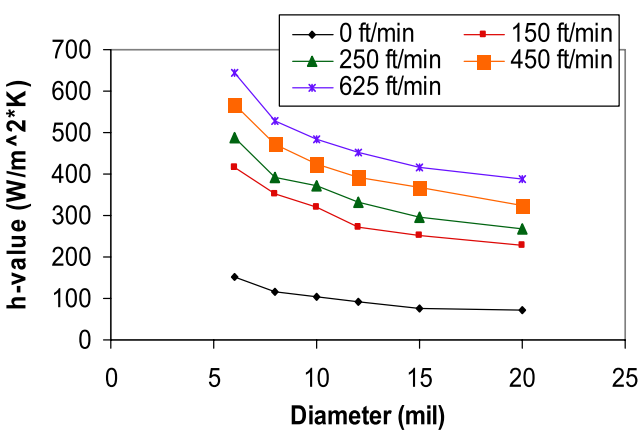

(b)

Figure 6. Effect of air flow rate on the convective coefficient wire diameter. Experimental data and three empirical fits are shown for $70{ }^{\circ} \mathrm{C}$ Flexinol SMA in air at $22{ }^{\circ} \mathrm{C}$. $h$-values decrease with increasing wire diameter, and increase with a decaying amount for increasing air flow rates, spanning a wide range of $h$-values. (a) Flow rate. (b) Diameter.

Table 4. Fit parameters for collected cooling data. Parameters for corrected fits are based on those suggested by Morgan [30] for free cooling and Hilpert [45] for forced cooling. Values based on experimental data are given for $C$ and $m$, along with an additional free variable $R$ for thermal grease.

\begin{tabular}{lllll}
\hline Medium & $\mathrm{Ra}_{D}$ & $C$ & $m$ & $R$ \\
\hline Still air & $10^{-10}-10^{-2}$ & 0.875 & 0.038 & \\
& $10^{-2}-10^{2}$ & 1.477 & 0.142 & \\
Moving air & $10^{-2}-10^{2}$ & 1.235 & 0.377 & \\
Mineral oil & $10^{-2}-10^{2}$ & 1.600 & 0.137 & \\
Thermal grease & & 2.998 & 0.130 & 10.3 \\
Water & $10^{-2}-10^{2}$ & 1.021 & 0.169 & \\
& $10^{2}-10^{4}$ & 0.868 & 0.208 & \\
\hline
\end{tabular}

3.2.2. Forced convection in air. When subjected to forced convection, the $h$ values for the SMA increased with flow rate but demonstrated a similar dependence on diameter as observed under free convection. For example, the results (figures 6(a) and (b)) show that the convective coefficient decreases by $40 \%$ under a fixed flow rate of $625 \mathrm{ft} \mathrm{min}^{-1}$ when the wire diameter is increased from 6 to 20 mil. This change in $h$ between different diameters also existed for other constant flow rates (see family of curves in figure 6(b)), decreasing on average by $47 \%$ with average deviations of $3 \%$. The family of curves in figure 6(a) shows the increasing trend with increasing flow rate, and heat transfer is improved under forced convection by an average factor of 4.9 (with $8 \%$ average deviation) for all wire diameters under a flow rate of $625 \mathrm{ft} \min ^{-1}$.

An equation of the form proposed by Hilpert [45] for horizontal cylinders under forced convection was used as a basis for constructing a continuous correlation for its relative simplicity. In this correlation, the Nusselt number is related to the Reynolds number, $\operatorname{Re}_{D}$, and Prandtl number, Pr, as

$$
\overline{\mathrm{Nu}}_{D}=\frac{\bar{h} D}{k}=C \operatorname{Re}_{D}^{m} \operatorname{Pr}^{1 / 3}
$$

where $C$ and $m$ are correlation constants (table 3 ) and $k$ is the thermal conductivity. The Reynolds number is defined as

$$
\operatorname{Re}_{D}=\frac{u D}{v}
$$

where $u$ is the flow rate, $D$ is the wire diameter in meters, and $v$ is the kinematic viscosity. For the data correlation, all fluid properties (table 3 ) were evaluated at an estimated mean film temperature of $42^{\circ} \mathrm{C}$, which is an average of the wire's surface temperature, $60^{\circ} \mathrm{C}$, and ambient temperature, $23^{\circ} \mathrm{C}$. As for the free cooling correlation, the mathematical limits on wire diameter of equation (5) match physical intuition. For values of $m$ less than 1 , the predicted $h$-value approaches infinity at very small diameters and zero at very large diameters.

Using the GRG algorithm, values of $C$ and $m$ (table 4) were optimized in equation (5) to minimize the average fit error to $6.4 \%$ for the range of data, capturing the effects of both diameter and flow rate. Figure 6(a) displays these fits (along with the unadjusted Hilpert fit) for the upper and lower bounding cases for wire diameter, and shows the adjusted fit over many wire diameters under an air flow rate of $625 \mathrm{ft} \mathrm{min}^{-1}$. For most data points, the predicted error was small but the model did deviate by as much as $13 \%$ for the maximum airflow speed of $625 \mathrm{ft} \mathrm{min}^{-1}$ for the 20 mil wire, though this is still a significant improvement over the $22 \%$ deviation from the unadjusted Hilpert correlation. These deviations may be due to a combination of the model's limitations in correlation and experimental error such as possible non-uniform airflow speeds.

\subsection{Free convection in mineral oil}

While increasing the air flow rate dramatically improves the convective heat transfer performance (figure 6(a)), higher frequency applications may warrant a change in medium since producing very high air flow rates may become impractical for reasons of fan size and power consumption. Mineral oil is an attractive alternative to air cooling due to its higher convective abilities and very high electrical resistance (greater than $10^{10} \Omega \mathrm{m}^{-1}$ [47]). To characterize mineral oil for its potential use in submersing SMA wires, experimental cooling data were collected for technical grade, light viscosity mineral oil obtained from McMaster-Carr, Inc. Figure 3 shows a similar trend to other media with respect to wire diameter as an increase in diameter generally reduces the heat transfer coefficient. The $h$-value reduction from 6 to $20 \mathrm{mil}$ is $49 \%$, which is close to the $44 \%$ reduction reported for 
free convection in air. In addition, when compared to free convection in air, the $h$-value for all diameters in mineral oil was consistently higher (e.g. the convection coefficient increased by a factor of 6.5 for 6 mil and 8.9 times for 20 mil). For the data correlation, the exact properties of the particular mineral oil used were not known, so values were estimated for general light viscosity oil [33]. The numbers are provided in table 2. A fit using these parameters was conducted with the GRG algorithm to determine values for $C$ and $m$ in the Hilpert correlation (equation (2)). Using the values provided in table 3 for mineral oil, the average fit error was minimized to only $3.8 \%$, which is an improvement upon the unadjusted average fit error of $36 \%$ (figure 3). Overall, mineral oil provides a higher increment in performance than $625 \mathrm{ft} \mathrm{min}^{-1}$ air flow, and as an added benefit requires no additional power draw. This, however, comes with a price of packaging complexity as the mineral oil must be sealed around the SMA wires. Additionally, the viscous nature of mineral oil can be problematic and cumbersome for parts that need frequent servicing.

\subsection{Effective convection in thermal grease}

To further improve the heat transfer from the SMA wire (beyond the performance of mineral oil) thermally conductive grease can be employed. Thermally conductive grease is typically used in the electronics industry to improve the thermal contact between a chip and a heat sink. For applications involving SMAs, it can be used in a similar fashion, the heated wire being immersed in the grease which acts as a direct conduit to transfer heat to an ambient environment. The experimental data shown in figure 3 represent the convective coefficient for various diameters of SMA immersed in OmegaTherm OT-201 thermally conductive silicon paste. The paste was selected due to its very high thermal conductivity, and also its electrically insulating properties. Unlike the rest of the fluids tested in this paper, the thermal grease is highly viscous and does not flow to produce free convection. Instead, the primary mode of heat transfer is conduction. For simplicity, and to allow for comparison with the other media tested, this form of heat transfer is approximated as an effective convective coefficient $h$. The data in figure 3 show that the effective convection is still heavily dependent on wire diameter. For example, the $h$-value decreases by $55 \%$ between 6 and 20 mil, which is of the same order as the decreases reported for air and mineral oil (44\% and $49 \%$, respectively). The amplitude of the effective $h$-values calculated for thermal grease is also significantly higher than for other media tested. For example, the effective convection at 6 mil wire diameter is 4.3 times higher than the same wire in mineral oil, and 28 times higher than free cooling in air.

A continuous fit to the collected data was again based on Morgan's free-convection equation (equation (2)), with the fluid properties lumped into an additional free variable $R$ (units of $\mathrm{s}^{2} \mathrm{~m}^{-4} \mathrm{~K}^{-1}$ ) since they were unknown. By substituting equation (3) into (2) and grouping terms the average convective coefficient is expressed as

$$
\bar{h}=C k\left[g R\left(T_{\mathrm{s}}-T_{\infty}\right)\right]^{m} D^{3 m-1}
$$

where the fluid properties are lumped into a single variable:

$$
R=\left(\frac{\beta}{v \alpha}\right)
$$

In the lumped correlation (equation (7)) the thermal conductivity $k$, was listed by the manufacturer to be $2.3 \mathrm{~W} \mathrm{~m}^{-1} \mathrm{~K}^{-1}$. Using the GRG optimization method, but allowing $R$ to vary in addition to $C$ and $m$, the average fit error was minimized to $3.1 \%$. The values of the optimized free variables are listed in table 4 , and the resulting fit is plotted with the data in figure 3. Because the grease was fitted to a fluid of lumped properties, the original Morgan correlation could not be calculated for comparison in this special case.

Compared to mineral oil, thermal grease provides significantly higher cooling allowing for higher frequency responses for a given diameter, which is important for many applications. It is also easier to seal since it does not flow through small spaces. However, thermal grease does have some disadvantages over mineral oil. For example, it is significantly more viscous and is consequently even more difficult to work with. Thermal grease is also expensive when compared to mineral oil and may become impractical when large quantities are needed.

\subsection{Free convection in water}

As long as the transition temperature for the SMA is below the boiling point of water, water immersion is an effective, yet simple, cooling method. For $70^{\circ} \mathrm{C}$ Flexinol wires, water also provides protection against overheating, since running excess current through the wires would cause the water to locally boil, dissipating the extra heat without damaging the wire. If the transition temperature of the wire is higher than the boiling temperature of pure water, ethylene glycol could be added to raise the fluid's boiling temperature (though this sacrifices some of water's convective properties). Because the transition temperatures of the tested $70^{\circ} \mathrm{C}$ Flexinol SMA wires were below the boiling point of pure water, the medium was tested with no additives. Additionally, to ensure that the medium's electrical resistance was very high (and that the input power measurements were accurate), technical grade distilled water from McMaster-Carr Inc. was used for the experiments.

The cooling performance for distilled water was tested to be the highest among all media studied (figure 3). When compared to the next highest tested medium, thermal grease, convective coefficients were approximately 1.3 times higher for 6 mil and 1.6 times higher for 20 mil wires when immersed in water. Compared to free cooling in air, performance is dramatically increased 36.5 and 44.5 times for 6 and 20 mil wire diameters, respectively. The strong dependence on wire diameter is also present in the water cooling data, with a $43 \%$ reduction in the convective coefficient from 6 to 20 mil. The data for free cooling were used to create a fit based on Morgan's correlation (equation (2)), and all fluid properties were readily found in the literature and are listed in table 2. Values for $C$ and $m$ were slightly adjusted by the GRG algorithm (values listed in table 4) to provide a continuous expression for the distilled water cooling with an average error of only $1.2 \%$, which is an 
improvement over the unadjusted Morgan's correlation error of $8 \%$. While this high cooling performance is excellent for high frequency applications, water cooling does increase system complexity since it must be sealed around the SMA. Additionally, if the high cooling performance of water is not needed it can actually reduce overall efficiency, producing significant losses during the heating cycle of the SMA.

\section{Conclusions}

This paper presents a new experimental method to measure the convective heat transfer coefficient from SMA wires based on the temperature-induced strain recovery of the SMA. By measuring the midpoint transformation strain as a function of temperature, it is possible to make predictions about surface temperature under various environmental conditions. These predictions enable Newton's cooling law to be employed to calculate the convective coefficient $h$ when the wire is supplied with a known power under steady-state conditions. Unlike traditional methods, this method is based on a strain measurement, allowing calculations of the convective coefficient to be made for any size wire in any ambient medium. This was demonstrated by measuring $h$ for wire diameters ranging from 6 to $20 \mathrm{mil}$ in air (free and flowing), mineral oil, thermally conductive grease, and water. To verify the midpoint transformation methodology, separate thermal imaging tests were also conducted for the only two feasible cases, 15 and 20 mil diameters in air. Close agreement between these two separate procedures was shown, demonstrating that the new transformation strain method is a robust procedure that overcomes measurement issues experienced by traditional techniques (i.e. the invasive properties of thermocouples on thin wires and problems with IR cameras viewing thin wires and used in media other than air).

The data collected using the transformation strain based method included environmental and geometric variations that produced a wide design space (spanning two orders of magnitude from $\sim 100$ to $\sim 5000 \mathrm{~W} \mathrm{~m}^{-2} \mathrm{~K}^{-1}$ ) from which an optimal combination of cooling medium and wire diameter can be selected based on cooling needs and power restrictions. As intuitively expected, increasing the wire diameter decreased the cooling performance for all cases (the average decrease in $h$ was $48 \%$ between 6 and 20 mil). The cooling medium also had a large effect as distilled water provided the highest $h$-value of all the tested media-between 3210 and $5590 \mathrm{~W} \mathrm{~m}^{-2} \mathrm{~K}^{-1}$ for the diameter range tested, which was as much as 1.6 times higher than that of conductive grease, and as much as 44.5 times higher than free convection in air. While providing very high heat transfer, water is more difficult to manage in an application than grease, requiring better sealing and special care for parts to resist corrosion, although conductive grease is significantly more expensive and is difficult to spread evenly throughout a device. Oil provided an intermediate level of $h$ value, between 512 and $997 \mathrm{~W} \mathrm{~m}^{-2} \mathrm{~K}^{-1}$, which was up to 7.1 times more than still air or up to 6.3 times less than water. Oil is inexpensive and does not promote corrosion, but does require careful sealing similar to water. Still air provided a lower $h$ value, between 69 and $153 \mathrm{~W} \mathrm{~m}^{-2} \mathrm{~K}^{-1}$, but is much easier and cheaper to use in an application requiring no additional parts or installation. Forced air, on the other hand, provided $h$-values between 389 and $644 \mathrm{~W} \mathrm{~m}^{-2} \mathrm{~K}^{-1}$ at a flow rate of $625 \mathrm{ft} \mathrm{min}^{-1}$, which was up to 5.6 times more than still air at the cost of requiring fans or some other means of blowing the air. An extra advantage gained by using forced convection is the ability to make live adjustments of the $h$-value over a large range (by a factor of 1.7 in these tests), such that the balance between cyclic performance and power consumption can be adjusted to a changing application. While not included in this study due to the additional complexities involved, forced convection of other fluids (water, in particular due to its low viscosity) can also be used to increase and tailor the $h$-value in situ, by potentially another order of magnitude as it did for air (figure 4).

Using the general form of proposed correlations in literature for the convection coefficient of a cylinder in still air and a cross-flow, empirical correlations were generated for each of the ambient media tested. Because the allencompassing fits found in the literature typically provided only general estimates of the collected data and did not apply well to wires of small diameter, the fit parameters were adjusted for each test condition using a GRG optimization algorithm. The resulting continuous equations closely followed the collected data in relating the power dissipation from the SMA wire to the wire diameter, flow rate, and fluid properties (as close as 1.2\% average error for water). The empirical correlations for all of the tested data can be used to extrapolate the wire diameter effect beyond the tested conditions if necessary. Because these correlations are continuous functions, they may readily be used in optimization algorithms to ensure that the cooling requirements are properly chosen to improve frequency performance while minimizing power consumption.

\section{References}

[1] Seelecke S and Müller I 2004 Shape memory alloy actuators in smart structures: modeling and simulation Appl. Mech. Rev. 5723

[2] Duerig T W, Melton K N, Stockel D and Wayman C M 1990 Engineering Aspects of Shape Memory Alloys (Northamptonshire, UK: Butterworth-Heinemann, Reed Books Services) p 499

[3] Huang W 2002 On the selection of shape memory alloys for actuators Mater. Design 23 11-9

[4] Chopra I 2002 Review of state of art of smart structures and integrated systems AIAA J. 40 2145-87

[5] Saadat S, Salichs J, Noori M, Hou Z, Davoodi H, Bar-On I, Suzuki Y and Masuda A 2002 An overview of vibration and seismic applications of NiTi shape memory alloy Smart Mater. Struct. 11 218-29

[6] Pathak A, Brei D, Luntz J, LaVigna C and Kwatny H 2007 Design and quasi-static characterization of SMASH: SMA stabilizing handgrip Proc. SPIE, Smart Structures and Materials vol 6523

[7] Pathak A, Brei D, Luntz J, LaVigna C and Kwatny H 2008 Dynamic characterization and single-frequency cancellation performance of SMASH (SMA actuated stabilizing handgrip) Proc. SPIE, Smart Structures and Materials vol 6926 
[8] McGavin G L and Guerin G 2002 Real-time seismic damping and frequency control of steel structures using nitinol wire Proc. SPIE, Smart Struct. Mater. 4696 176-84

[9] Epps J J and Chopra I 2001 In-flight tracking of helicopter rotor blades using shape memory alloy actuators Smart Mater. Struct. 10 104-11

[10] Sanders B, Cowan D and Scherer L 2004 Aerodynamic performance of the smart wing control effectors J. Intell. Mater. Syst. Struct. 15293

[11] Pitt D M et al 2001 Wind tunnel demonstration of the SAMPSON smart inlet Proc. SPIE, Smart Struct. Mater. 4332 345-56

[12] Vaidyanathan R, Chiel H J and Quinn R D 2000 A hydrostatic robot for marine applications Robot. Auton. Syst. 30 103-13

[13] Bertetto A M and Ruggiu M 2003 A two degree of freedom gripper actuated by SMA with flexure hinges J. Robot. Syst. 20 649-57

[14] Dilibal S, Guner E and Akturk N 2002 Three-finger SMA robot hand and its practical analysis Robotica 20 175-80

[15] DeLaurentis K J, Mavroidis C and Pfeiffer C 2000

Development of a shape memory alloy actuated robotic hand Proc. ACTUATOR 2000 Conf. (Bremen, June 2000) pp 281-5

[16] Makino E, Mitsuya T and Shibata T 2001 Fabrication of TiNi shape memory micropump Sensors Actuators A 88 256-62

[17] Xu D, Wang L, Ding G, Zhou Y, Yu A and Cai B 2001 Characteristics and fabrication of NiTi/Si diaphragm micropump Sensors Actuators A 93 87-92

[18] Kohl M, Dittmann D, Quandt E and Winzek B 2000 Thin film shape memory microvalves with adjustable operation temperature Sensors Actuators A $83214-9$

[19] Ho K K et al 1999 Fabrication and characterization of thin film NiTi for use as a microbubble for active flow control Proc. 6th Ann. Int. Symp. Smart Structures and Materials (Newport Beach, CA, March 1999) ed M Wuttig (Bellingham, WA: SPIE Optical Engineering Press)

[20] Huang W M, Tan J P, Gao X Y and Yeo J H 2003 Design, testing, and simulation of NiTi shape-memory-alloy thin-film-based microgrippers J. Microlithogr. Microfabrication Microsyst. 2185

[21] Zhang H, Bellouard Y, Sidler T C, Burdet E, Poo A N and Clavel R 2001 Monolithic shape memory alloy microgripper for 3D assembly of tissue engineering scaffolds Proc. SPIE, Smart Structures and Materials (Boston, MA)

[22] Krulevitch P, Lee A P, Ramsey P B, Trevino J C, Hamilton J and Northrup M A 1996 Thin film shape memory alloy microactuators J. Microelectromech. Syst. 5 270-82

[23] Roch I, Bidaud P, Collard D and Buchaillot L 2003 Fabrication and characterization of an SU-8 gripper actuated by a shape memory alloy thin film J. Micromech. Microeng. 13 330-6

[24] Winzek B, Schmitz S, Rumpf H, Sterzl T, Hassdorf R, Thienhaus S, Feydt J, Moske M and Quandt E 2004 Recent developments in shape memory thin film technology Mater. Sci. Eng. A 378 40-6

[25] Shaw J A 2002 A thermomechanical model for a 1D shape memory alloy wire with propagating instabilities Int. J. Solids Struct. 39 1275-305

[26] Barnes B M, Brei D E, Luntz J E, Strom K, Browne A L and Johnson N 2008 Shape memory alloy resetable spring lift for pedestrian protection Proc. SPIE, Smart Structures and Materials (San Diego, CA)

[27] Potapov P L and Silva E P D 2000 Time response of shape memory alloy actuators J. Intell. Mater. Syst. Struct. 11125
[28] Shahin A R, Meckl P H, Jones J D and Thrasher M A 1994 Enhanced cooling of shape memory alloy wires using semiconductor 'heat pump' modules J. Intell. Mater. Syst. Struct. 595

[29] Bhattacharyya A, Sweeney L and Faulkner M G 2002 Experimental characterization of free convection during thermal phase transformations in shape memory alloy wires Smart Mater. Struct. 11 411-22

[30] Morgan V T 1975 The overall convective heat transfer from smooth circular cylinders Adv. Heat Transfer 11 199-264

[31] Zukauskas A, Ziugzda J and Hewitt G F 1985 Heat Transfer of a Cylinder in Crossflow (Washington, DC: Hemisphere)

[32] Sanitjai S and Goldstein R J 2004 Forced convection heat transfer from a circular cylinder in crossflow to air and liquids Int. J. Heat Mass Transfer 47 4795-805

[33] Incropera F P and DeWitt D P 2001 Heat and Mass Transfer (New York: Wiley)

[34] Omega Engineering, Inc 2009 cited; available from: http://www.omega.com

[35] Wu X D, Fan Y Z and Wu J S 2000 A study on the variations of the electrical resistance for NiTi shape memory alloy wires during the thermo-mechanical loading Mater. Design 21 511-5

[36] Nam T H, Noh J P, Jung D W, Kim Y W, Im H J, Ahn J S and Mitani T 2002 The R phase transformation in Ti-49Ni (at.\%) shape memory alloy ribbons fabricated by melt spinning J. Mater. Sci. Lett. 21 11-3

[37] Churchill C B and Shaw J A 2008 Shakedown response of conditioned shape memory alloy wire Proc. SPIE, Smart Structures and Materials (San Diego, CA)

[38] Ay H, Jang J Y and Yeh J N 2002 Local heat transfer measurements of plate finned-tube heat exchangers by infrared thermography Int. J. Heat Mass Transfer 45 4069-78

[39] Electrophysics Corp 2008 cited; available from: http://www. electrophysics.com

[40] Kandlikar S G, Joshi S and Tian S 2001 Effect of channel roughness on heat transfer and fluid flow characteristics at low Reynolds numbers in small diameter tubes Atmosphere 47

[41] Koo J and Kleinstreuer C 2005 Analysis of surface roughness effects on heat transfer in micro-conduits Int. J. Heat Mass Transfer 48 2625-34

[42] Pohl M, Heßing C and Frenzel J 2004 Electrolytic processing of NiTi shape memory alloys Mater. Sci. Eng. A 378 191-9

[43] Sun H, Pathak A, Luntz J, Brei D, Alexander P W and Johnson N L 2008 Stabilizing shape memory alloy actuator performance through cyclic shakedown: an empirical study Proc. SPIE, Smart Structures and Materials (San Diego, CA)

[44] Meinders E R, Van Der Meer T H and Hanjalic K 1998 Local convective heat transfer from an array of wall-mounted cubes Int. J. Heat Mass Transfer 41 335-46

[45] Hilpert R 1933 Correlations for laminar forced convection in flow over an isothermal flat plate and in developing and fully developed flow in an isothermal tube Forsch. Geb. Ing. 4 215-24

[46] McQuillan F 2009 Thermal Fluid Properties Calculator cited; available from: http://www.mhtl.uwaterloo.ca/properties. html

[47] 2008 Tables of Physical and Chemical Constants cited; available from: http://www.kayelaby.npl.co.uk 